\title{
Sorafenib Induced Hand and Foot Syndrome
}

\author{
Dhanya Susan' ${ }^{1}$ Malini $\mathbf{M},{ }^{2 *}$ Remya Reghu \\ 'Pharm D intern, Department of Pharmacy Practice, Amrita School of Pharmacy, Kochi 682041, Amrita University, INDIA. \\ ${ }^{2}$ Assistant Professor, Department of Pharmacy Practice, Amrita School of Pharmacy, Kochi 682041, Amrita University, INDIA.
}

\begin{abstract}
Sorafenib a multikinase inhibitor has been approved by the FDA for the treatment of thyroid, hepatocellular and renal cell carcinoma. Hand-foot syndrome / palmar - plantar erythrodysesthesia causes redness, swelling and pain on the palms of the hands and / or the soles of the feet. Sometimes blisters also appear. Here, we report a post right hepatectomy hepatocellular carcinoma patient who developed hand and foot syndrome after a dose with sorafenib.
\end{abstract}

Key words: Sorafenib, Hand and Foot Syndrome, Multikinase Inhibitor,
Hepatocellular, Carcinoma.

Correspondence

Remya Reghu, Assistant Professor, Department of Pharmacy Practice, Amrita School of Pharmacy, Kochi 682041, Amrita University, INDIA.

Phone: + 91984788184

Email: remyareghu@aims.amrita.edu

DOI: 10.5530/jyp.2018.10.29

\section{INTRODUCTION}

Sorafenib has been approved by the FDA for the treatment of primary renal cell carcinoma, thyroid carcinoma and hepatocellular carcinoma. ${ }^{1-8}$ It is a multikinase inhibitor (tyrosine kinase, Raf serine / threonine kinases). It also inhibits vascular endothelial growth factor (VEGF), platelet - derived growth factor $\beta$ (PDGF $\beta$ ), and tumour progression. ${ }^{2,3-9}$ Adverse reactions to sorefinib are gastrointestinal (diarrhoea, increased amylase and lipase, nausea, constipation), dermatological (acne, flushing, rash / desquamation, hand - foot syndrome (HFD), alopecia, pruritus), hyperthyroidism, hypertension and hypoalbuminemia. ${ }^{1}$ HFD is characterized by reddening, swelling, numbness and desquamation on palms and soles. In this case report, we describe a case of HFD caused by sorafenib.

\section{METHOD}

The case report is of a 71 year old lady with a known case of diabetes mellitus (type II), peripheral neuropathy on Metformin $500 \mathrm{mg} \mathrm{BD}$ and H.Mixtard 30-0-20 was diagnosed with hepatocellular carcinoma (grade III) with IVC tumor thrombus and underwent Right hepactectomy. She was started on Sorafenib $200 \mathrm{mg}$ once daily.

While on therapy after 14 days she complained of loose stools with a frequency up to 5 times / day, which was managed conservatively. Since the patient was found to be tolerating, the dose of Sorafenib was hiked up to $200 \mathrm{mg}$ twice daily. Later on after two months she developed toe nail ulcers, paronychia, oral ulcers, diarrhea, all probable side effects of sorafenib.

Since last 5 days she again presented with multiple abscesses over her plantar region of right and left foot (fig. 1). Since she was on sorafenib and with a history of previous similar lesions, a possibility of sorafenib induced hand foot syndrome was suspected.

Upon investigation, her blood counts were normal with mildly elevated inflammatory markers (CRP $25.7 \mathrm{mg} / \mathrm{L}$ ).

She was managed conservatively with intravenous Ampicillin and Cefoperazone sulbactam. She was also advised with off-loading and proper foot care. Insulin doses were adjusted to maintain euglycemia. Repeat blood counts were stable and iv antibiotics were stopped after 7 days, Symptomatic improvement was noted. Further Medical oncology consultation was sought and planned to stop sorafenib.

Causality assessment was carried out using the Naranjo's scale. The algorithms showed that sorafenib was the "definite" (Naranjo's score 9) cause of this adverse drug reaction.

\section{DISCUSSION}

Sorafenib induced hand and foot syndrome has been reported in patients with breast cancer, melanomas, renal cell cancer and hepatocllular cancers. ${ }^{4,5,6}$ The incidence of hand and foot syndrome is $25-30 \%$ of patients who are on standard dose i.e; $400 \mathrm{mg}$ twice daily and is found to be well tolerated. Sorafenib is also associated with other dermal changes such as alopecia, pruritis, nail changes, flushing etc.

Compared to the hand and foot syndrome caused by cytarabine, 5 FFU and MTX, the one caused by sorafenib though it is indistinguishable, it is less severe, more localized and affects friction and weight bearing acral surfaces. ${ }^{1}$ The HFD due to sorafenib is usually dose dependent and the dose is usually resorted for the abetment of the symptoms. ${ }^{1}$ In this present case the laboratory parameters were normal in range once the drug was stopped. Thus, we can label the case as sorafenib induced hand and foot syndrome.

Prevention of HFD can be made by reducing the exposure of hands and feet to hot water, excessive rubbing, and applying moisturizing cream, exfoliating the hyperkeratosis palms and soles. ${ }^{6}$

Various possible mechanism has been evaluated for sorafenib induced hand and foot syndrome, among which the anti- VEGF property has been hypothesized to be the probable pathogenesis. ${ }^{7}$ Sorafenib is been widely found to be effective for various solid tumors, hence as its use increases the need for high index suspicion is warranted for the prevention, early detection and treatment of HFD. 


\section{CONFLICTING INTEREST}

The author(s) declared no potential conflicts of interest with respect to the research, authorship, and/or publication of this article.

\section{REFERENCES}

1. Sil A, Das NK. Sorafenib-induced hand-foot syndrome in a patient of renal cell carcinoma. Indian J Pharmacol 2014;46(3):334-6.

2. Lacouture ME, Reilly LM, Gerami P, Guitart J. Hand foot skin reaction in cancer patients treated with the multikinase inhibitors sorafenib and sunitinib. Annals of Oncology. 2008;19(11):1955-61.

3. Robert C, Mateus C, Spatz A, Wechsler J, Escudier B. Dermatologic symptoms associated with the multikinase inhibitor sorafenib. Journal of the American Academy of Dermatology. 2009;60(2):299-305.

4. Gomez P, Lacouture ME. Clinical presentation and management of hand-foot skin reaction associated with sorafenib in combination with cytotoxic chemotherapy: experience in breast cancer. The oncologist. 2011;16(11):1508-19.

5. Lee JH, Chung YH, Kim JA, Shim JH, Lee D, Lee HC, et al. Genetic predisposition of hand-foot skin reaction after sorafenib therapy in patients with hepatocellular carcinoma. Cancer. 2013;119(1):136-42.

6. Lountzis NI, Maroon MS. Sorafenib-induced palmoplantar hyperkeratosis. Journal of drugs in dermatology: JDD. 2008;7(6):588-9.

7. Azad NS, Aragon-Ching JB, Dahut WL, Gutierrez M, Figg WD, Jain L, et al. Hand-foot skin reaction increases with cumulative sorafenib dose and with combination anti-vascular endothelial growth factor therapy. Clinical Cancer Research. 2009;15(4):1411-6.

8. Pooleri GK, Nair TB, Sanjeevan KV, Thomas A. Neo Adjuvant Treatment with Targeted Molecules for Renal Cell Cancer in Current Clinical Practise. Indian journal of surgical oncology. 2012;3(2):114-9.

9. Palaniappan M, Jose WM, Mehta A, Kumar K, Pavithran K. Umbilical metastasis: a case series of four Sister Joseph nodules from four different visceral malignancies. Current Oncology. 2010;17(6):78-81

Article History: Submission Date : 06-06-2017 ; Revised Date : 21-07-2017; Acceptance Date : 31-08-2017.

Cite this article: Susan D, Malini M, Reghu R. Sorafenib Induced Hand and Foot Syndrome. J Young Pharm. 2018;10(1):129-30. 